OPEN

SUBJECT AREAS:

ATOMIC AND

MOLECULAR PHYSICS

CONDENSED-MATTER PHYSICS

OPTICAL PHYSICS

ULTRACOLD GASES

Received

6 September 2012

Accepted

23 October 2012

Published

7 January 2013

Correspondence and requests for materials should be addressed to

W.T.H. (wth@umd. edu)

\section{Analogs of Basic Electronic Circuit Elements in a Free-Space Atom Chip}

\author{
Jeffrey G. Lee, Brian J. Mcllvain, C. J. Lobb \& W. T. Hill, III \\ Joint Quantum Institute, University of Maryland, College Park, MD 20742 USA.
}

Using a thermal sample of laser-cooled rubidium atoms, we have constructed a neutral-atom circuit analogous to an electronic capacitor discharged through a resistor. The atoms are confined using what we call a free-space atom chip, an optical dipole trap created using a generalized phase-contrast imaging technique. We have also calculated theoretical values for the capacitance and resistance, which agree with our experiments, as well as theoretical value for an atomic analog of electrical inductance. We show that atomic capacitance is analogous to the quantum capacitance, the atomic resistance is analogous to the ballistic, or Sharvin resistance, and the atomic inductance is analogous to kinetic inductance.

lectronics is based on the manipulation of electrons, possibly enhanced by exploiting internal structure associated with spin (spintronics). Atomtronics seeks do the same with neutral atoms. The more complex internal structure of atoms makes the possibilities of such an architecture far richer than its electronic analog. Recently, persistent currents of superfluid neutral atoms have been created in ring circuits, which are close analogs to superconducting circuits ${ }^{1}$. There have also been efforts towards developing analogs to active electronic circuit elements such as diodes and transistors ${ }^{2}$. Any usable neutral atom circuit, however, will need analogs to the most basic electronic elements - resistors $(R)$, capacitors $(C)$ and inductors $(L)$. Using a two-dimensional (2D) optical dipole potential ${ }^{3}$ to generate what we call free-space atom chips, we have created and characterized a neutral-atom $R L C$ circuit. We show that the resistance is analogous to ballistic (Sharvin ${ }^{4}$ ) resistance in metals, the inductance is analogous to kinetic inductance in superconductors ${ }^{5}$, and the capacitance is analogous to the quantum capacitance in nanoscale devices ${ }^{6,7}$.

A $2 \mathrm{D}$ neutral atom analog to an electronic $R L C$ circuit can be realized with a classical ideal gas using two containers of areas $A_{1}$ and $A_{2}$ connected by a channel. In an electronic capacitor, a charge imbalance $(Q)$ between two conductors produces a potential difference $(\Delta V)$. When a resistor connects the two conductors, electrons flow to eliminate the charge imbalance, causing $\Delta V$ to vanish. In our system, a number imbalance $(N)$ between the two containers produces a chemical potential difference $(\Delta \mu)$. When a channel connects the two containers, atoms flow to eliminate the number imbalance, causing $\Delta \mu$ to vanish. To complete this analogy, we define a chemical capacitance,

$$
C_{c}=\frac{N}{\Delta \mu}
$$

with SI units of $1 / \mathrm{J}$ and with the same form as the electronic capacitance $C=Q / \Delta V$.

The chemical potential of a $2 \mathrm{D}$ ideal gas is the change in free energy associated with changing the number of particles in the system ${ }^{8}$,

$$
\mu=k T \ln \left[n\left(\frac{h^{2}}{2 \pi m k T}\right)\right],
$$

where $k$ is Boltzmann's constant, $h$ Planck's constant, $T$ the temperature, $n$ the $2 \mathrm{D}$ number density and $m$ the mass of a particle in the gas. To determine the capacitance, we start by assuming the two chambers to be in equilibrium, with equal temperatures and equal densities, $N_{1 e} / A_{1}=N_{2 e} / A_{2}=n_{e}$. Moving a small number of particles $N$ from $A_{1}$ to $A_{2}$ leads to an imbalance in the densities so that $n_{1}=\left(N_{1 e}-N\right) / A_{1}$ and $n_{2}=\left(N_{2 e}+N\right) / A_{2}$. Subtracting equation (2) with $n_{1}$ from equation (2) with $n_{2}$ leads to

$$
\begin{aligned}
\Delta \mu & =k T \ln \left(\frac{n_{2}}{n_{1}}\right)=k T\left\{\ln \left[\left(\frac{N_{2 e}}{A_{2}}\right)\left(1+\frac{N}{N_{2 e}}\right)\right]-\ln \left[\left(\frac{N_{1 e}}{A_{1}}\right)\left(1-\frac{N}{N_{1 e}}\right)\right]\right\} \\
& \approx k T\left(\frac{A_{1}+A_{2}}{A_{1} A_{2}}\right)\left(\frac{N}{n_{e}}\right) .
\end{aligned}
$$


This linear approximation is valid for $N \ll N_{1 e}$ and $N_{2 e}$. The experiment we describe below is within this limit. Combining equations (1) and (4) leads to an expression for the neutral-atom chemical capacitance,

$$
C_{c}=\left(\frac{n_{e}}{k T}\right)\left(\frac{A_{1} A_{2}}{A_{1}+A_{2}}\right) \equiv \frac{n_{e} \bar{A}}{2 k T}=\frac{n_{e} \bar{A}}{2 \varepsilon_{T}},
$$

where $\bar{A}$ is the harmonic mean of $A_{1}$ and $A_{2}$, and $\varepsilon_{T}$ is the thermal energy of the gas.

When a channel is opened between the chambers, it acts as a resistor and an inductor. To determine the values of $R$ and $L$, we examine the flow dynamics of the system. In a $2 \mathrm{D}$ ideal gas, the effusion rate $F$ of atoms out of either container is given by

$$
F_{i}=\sqrt{\frac{k T}{2 \pi m}} \frac{N_{i}}{A_{i}} w,
$$

where $A_{i}$ is the area of the container and $w$ is the width of the channel. The total rate of change in atom number in container 1 can then be written as

$$
\dot{N}_{1}(t+\Delta t)=-F_{1}(t+\Delta t)+F_{2}(t),
$$

where the second term on the right hand side of the equation, representing the rate that atoms enter container 1 due to leaving container 2 , is evaluated at a time $\Delta t$ earlier. This delay is due to the finite velocity of the atoms traversing the channel. We approximate this delay time using the average transverse velocity of an atom in the channel,

$$
\langle\Delta t\rangle=\frac{l}{\left\langle v_{x}\right\rangle}=l \sqrt{\frac{2 m}{\pi k T}},
$$

where $l$ is the length of the channel. When we combine equations (58 ), and linearly expand the terms in equation (7) involving $\Delta t$, the result is analogous to Kirchhoff s law applied to a series $R L C$ circuit,

$$
\frac{N}{C_{c}}=-R_{c} \dot{N}-L_{c} \ddot{N},
$$

with chemical resistance and inductance given by

$$
R_{c}=\frac{2\left\langle p_{2 D}\right\rangle}{n_{e} w} \quad \text { and } \quad L_{c}=\frac{2 m l}{n_{e} w},
$$

Table 1 | Summary of chemical capacitances and resistances for various systems. In the $2 \mathrm{D}$ systems, $\bar{A}$ is the harmonic mean of the two container areas, $w$ is the cross-sectional width of the channel, the average classical $2 D$ momentum is $\left\langle p_{2 D}\right\rangle \sqrt{\pi m k T / 2}$, and

\begin{tabular}{|c|c|c|c|c|}
\hline & \multicolumn{2}{|c|}{$C_{c}$} & \multicolumn{2}{|c|}{$R_{c}$} \\
\hline & $2 \mathrm{D}$ & $3 D$ & $2 D$ & $3 D$ \\
\hline \multirow{2}{*}{ Classical } & 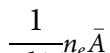 & \multirow{2}{*}{$\frac{1}{2 k T} n_{e} \bar{V}$} & $2\left\langle p_{2 D}\right\rangle$ & $\pi\left\langle p_{3 D}\right\rangle$ \\
\hline & $2 k T^{2+2 t}$ & & $n_{e} w$ & $2 n_{e} a$ \\
\hline & $m \bar{A}$ & $\left(3 n_{p}\right)^{1 / 3} \mathrm{~m}$ & $\pi p_{F_{2 D}}$ & $4 p_{F_{3 D}}$ \\
\hline Fermi $T=0$ & $2 \pi \hbar^{2}$ & $\left(\frac{m_{e}}{\pi}\right) \quad \frac{\pi}{2 \pi \hbar^{2}} \bar{V}$ & $\overline{2} \overline{n_{e} w}$ & $\overline{3} \overline{n_{e} a}$ \\
\hline \multirow{2}{*}{ BEC (GP) } & $\bar{A}$ & $\bar{V}$ & & \\
\hline & $\overline{2 g}$ & $\overline{2 g}$ & & \\
\hline
\end{tabular}
the 2D Fermi momentum is $p_{F_{2 D}}=\hbar \sqrt{2 \pi n_{e}}$. In the 3D systems, $\bar{V}$ is the harmonic mean of the two container volumes, $a$ is the cross-sectional area of the channel, the average classical 3D momentum is $\left\langle p_{3 D}\right\rangle=\sqrt{8 m k T / \pi}$, and the 3D Fermi momentum is $p_{F_{3 D}}=\hbar \sqrt[3]{3 \pi^{2} n_{e}}$ with SI units of $\mathrm{J} \cdot \mathrm{s}$ and $\mathrm{J} \cdot \mathrm{s}^{2}$ respectively. Here,

$$
\left\langle p_{2 D}\right\rangle=\sqrt{\frac{\pi m k T}{2}}
$$

is the average $2 \mathrm{D}$ momentum of the particles in one container. We dropped a second term in the resistance that is only significant when the channel represents a large portion of the entire area of the system. At this point the small $\mathrm{N}$ approximation breaks down, thus the second term is negligible when equation (9) is valid.

The chemical capacitance, resistance and inductance each have specific electronic analogs. Our capacitance results from the shift in chemical potential due to transfer of particles, the same effect that causes the quantum capacitance in nanostructures ${ }^{6,7}$. For 2D quantum dots with areas $A_{1}$ and $A_{2}$, the quantum capacitance is given by

$$
C_{q}=\frac{e^{2} m \bar{A}}{2 \pi \hbar^{2}}=\frac{e^{2} n_{e} \bar{A}}{2 \varepsilon_{F}}
$$

where $\varepsilon_{F}$ is the Fermi energy of the gas. This is similar to equation (5), with an electrical factor of $e^{2}$ and the thermal energy replaced by the $2 \mathrm{D}$ Fermi energy. It is interesting to note that taking $\hbar \rightarrow 0$ in equation (12) does not yield the correct classical limit of the quantum capacitance, which is equation (5).

In electronics, a point junction smaller than the mean free path of the electrons creates what is known as the Sharvin resistance. This arises purely from the ballistic motion of the electrons because there is no scattering of electrons in the point junction itself. For a $2 \mathrm{D}$ electronic system the Sharvin resistance is given by ${ }^{9}$

$$
R_{S}=\frac{\pi}{2} \frac{p_{F}}{e^{2} n w},
$$

where $p_{F}=\hbar \sqrt{2 \pi n_{e}}$ is the Fermi momentum. This has the same form as our chemical resistance in equation (10), differing by a factor of $e^{2}$ and a numerical factor because the Sharvin resistance is derived for a 2D, charged, $T=0$ Fermi gas, which has a different velocity distribution than our neutral, classical gas.

There is an inductance in electronic systems, the kinetic inductance $^{5}$, that arises due to the kinetic energy associated with current flow in a wire,

$$
L_{k}=\frac{m l}{e^{2} n w}
$$

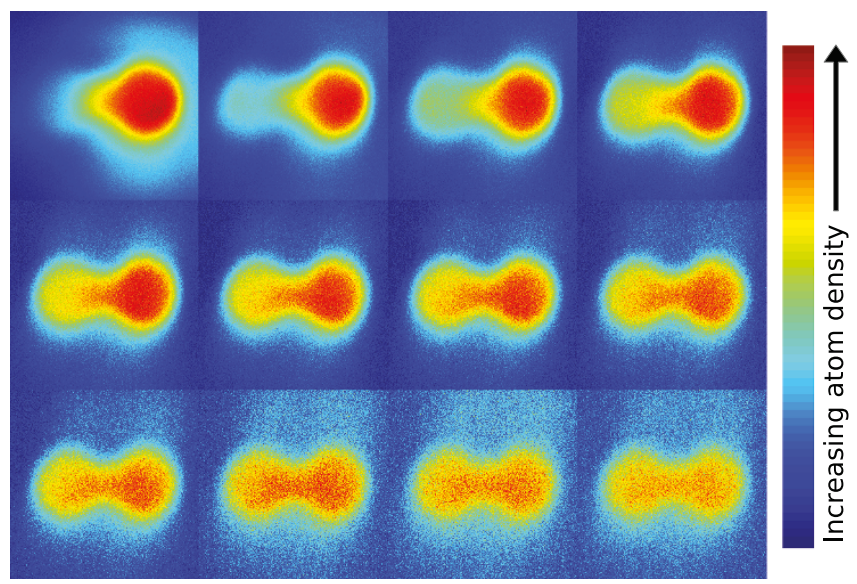

Figure 1 Capacitor Discharge. Images of a discharging atom capacitor with a channel width of $340 \mu \mathrm{m}$. The atoms are loaded into the right container and released from the MOT at $t=0 \mathrm{~ms}$. The images are snapshots at $5 \mathrm{~ms}$ intervals, starting at $t=5 \mathrm{~ms}$ (viewed left to right, top to bottom). Each frame is the average of eight individual experimental runs, and is scaled independently of the other frames. 


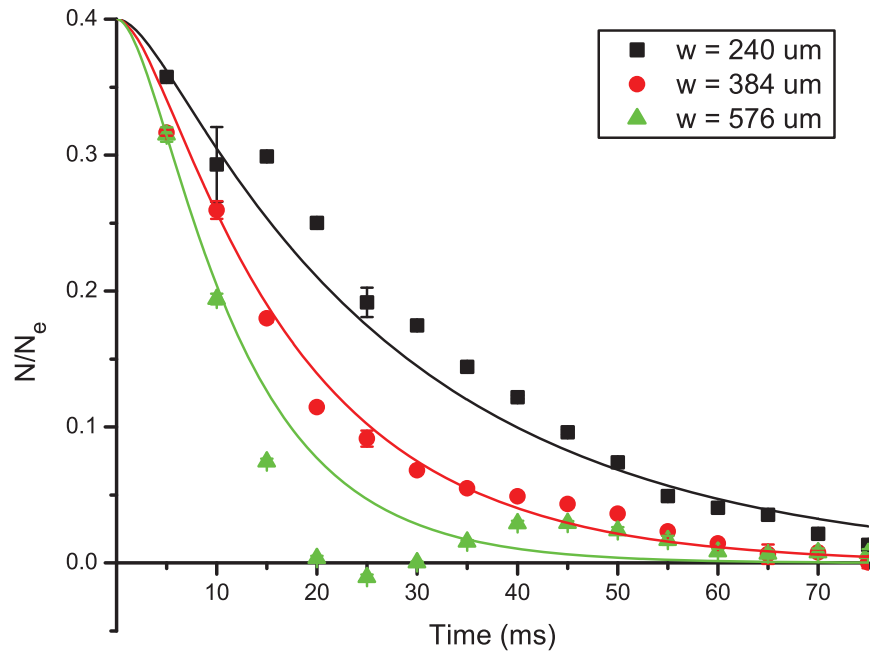

Figure 2 Comparison of experimental data to analytic solution. The points indicate the experimental data and the solid lines represent the relevant $R L C$ solution. The black line is for a channel width of $240 \mu \mathrm{m}$, the red $384 \mu \mathrm{m}$, and the green $576 \mu \mathrm{m}$. From equations $(5,10)$, the RC time constant, $\tau=\sqrt{\frac{\pi m}{2 k T}} \frac{A}{w}$, can be calculated for each of these systems to be 34, 21 and $14 \mathrm{~ms}$ respectively. The diameter of each container is $600 \mu \mathrm{m}$. For each experimental data point, 8 individual runs were averaged. The error bars are the standard deviation of the mean.

Again, this shares the same form as our chemical inductance, differing by a factor of $e^{2}$ and a numerical factor.

The methods that we used to calculate the capacitance and resistance of classical ideal gas circuit elements can be extended to other atomic systems such as degenerate Fermi gases and Bose-Einstein condensates. To define the chemical capacitance, all that is needed is the dependence of the chemical potential on atom number. The momentum distribution of the sample determines the effusion rate, which leads directly to the chemical resistance. Table 1 summarizes these results for $2 \mathrm{D}$ and $3 \mathrm{D}$ non-interacting classical gases, noninteracting degenerate Fermi gases, and BECs. The BEC cases are derived using the Gross-Pitaevskii (GP) equations for hard-wall containers in the Thomas-Fermi limit. In this case it is not sufficient to describe the system as consisting of point particles with classical trajectories, which is needed to derive the resistance, thus this is omitted. The 2D Fermi gas capacitance formula is exact. The classical gas and the 3D Fermi gas capacitances are all the first-order terms in Taylor series expansions.

\section{Results}

To realize a classical ideal gas neutral atom capacitor, we begin with a sample of ${ }^{87} \mathrm{Rb}$ atoms, cooled and confined using a magneto-optical $\operatorname{trap}^{10}$ (MOT). This MOT is created inside of a free-space atom chip that consists of two circular containers separated by a rectangular channel. The method used for creating this potential is discussed in the methods section. At time $t=0$, the atoms are released from the MOT after a final cooling stage, and allowed to flow between the containers for a variable amount of time, after which a fluorescence image is taken of the atoms. We directly image the gas flow between the containers with this setup. The evolution of this system can be seen in figure 1. We point out that the arrangement employed is similar to that used in atom-optics billiards where a circular potential is known to lead to "regular" dynamics, which would affect the effusion rate in our experiment ${ }^{11}$. In our case however, the container walls are not smooth, as discussed in the methods. Because of this the atom-wall collisions are not always specular, leading to ergodic dynamics, thus our use of equation (6) is valid. Figure 2 shows the decay of the normalized number imbalance $N / N_{e}$ as a function of time for several channel widths, as well as the solution to the $R L C$ differential equation for the parameters used. The only fit parameter for the $R L C$ solution is the initial atom number imbalance.

\section{Discussion}

For channels up to approximately half the width of the containers, the data and theory agree well. For channels that approach the width of the containers (green plot in figure 2), the assumption of local equilibrium is no longer valid, and good agreement is not expected, and indeed is not seen. The nature of the disagreement appears to be inductive, showing a significant overshoot. This however, is not the inductance we derive, which is too small to account for this phenomenon.

We have experimentally demonstrated atomtronic capacitors and resistors, and shown how to construct an atomtronic inductor, all on a free-space atom chip defined using $2 \mathrm{D}$ optical dipole potentials. These basic linear devices are necessary for biasing active devices, and are also essential for constructing oscillators and filters. Integration into systems such as loops containing $\mathrm{BECs}^{1}$, atomtronic Josephson junctions based on BECs, BEC analogs of SQUIDs ${ }^{12}$, and atomtronic batteries, diodes and transistors ${ }^{2}$, is made much more simple through the flexibility of our atom-chip technique.

\section{Methods}

To create the trap geometry that was necessary to carry out this experiment, we used what we call free-space atom chips, which are a type of crossed optical dipole trap. We use a sheet dipole trap to confine the atoms to a $2 \mathrm{D}$ plane. On top of this, we project an arbitrary $2 \mathrm{D}$ optical dipole potential to create the geometry necessary for the experiment. In this particular experiment, the sheet trap was a blue-detuned, repulsive beam confining the atoms from below, with gravity confining them from above.

In the plane, we create our arbitrary $2 \mathrm{D}$ pattern using a generalized phase contrast approach $^{13}$. This scheme is shown in figure $3 \mathrm{a}$ ). A phase pattern is imprinted onto an input TEM ${ }_{00}$ beam and sent through a 4 -f imaging system. We used a $2 \mathrm{D}$ spatial light modulator (SLM) to imprint the pattern. At the Fourier plane, there is a phasecontrast filter that shifts the phase of the lowest spatial frequencies, which exist near the optical axis, by $\pi$ relative to the remaining spatial frequencies of the beam. The two parts of the beam interfere in the output plane to produce an intensity profile that mirrors the input phase mask. This plane is imaged onto the sheet potential, effectively etching the sheet to create our free-space atom chip. The intensity profile for one of our capacitor potentials is shown in figure $3 \mathrm{~b}$ ). This method, with the use of the computer-addressable SLM, allows us to create and easily modify the confining potential used in the experiment. This ability was integral to this experiment as it
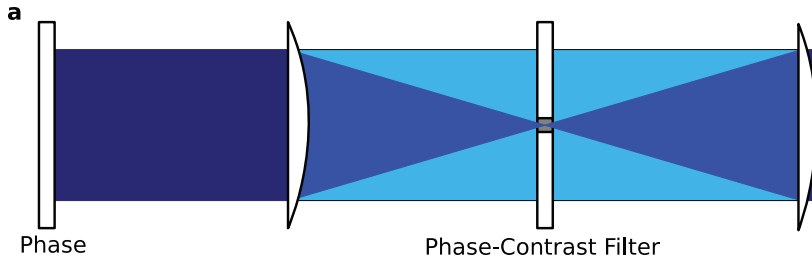

Phase-Contrast Filter

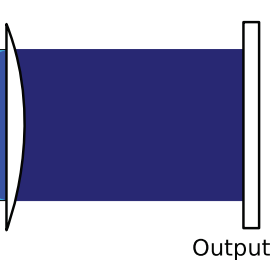

b

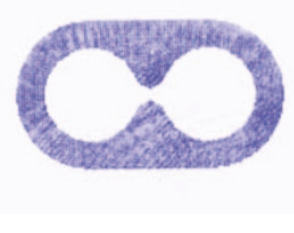

Figure 3 Phase-Contrast imaging system with typical output. a) A basic phase-contrast imaging system with input phase mask, phase-contrast filter, and 4 -f lens arrangement, and b) a typical output pattern used to create blue-detuned optical dipole potentials for an atom capacitor. The blue (white) areas correspond to light (absence of light). 
allowed us to easily adjust the channel width. Because of the pixelazation of our SLM, the walls of our containers are rough on the order of $10 \mu \mathrm{m}$.

In the experiment, the atoms start with a temperature of approximately $40 \mu \mathrm{K}$. The potential height of the container walls is approximately $60 \mu \mathrm{K}$. Since there is a significant population of atoms in the thermal distribution with an energy that can escape the trap, there is a truncated velocity distribution in the sample. The number density of the atoms in this experiment is not high enough for rethermalization to occur. Timeof-flight expansion experiments have shown that the truncated distribution is well approximated by a thermal distribution at a temperature of about $20 \mu \mathrm{K}$, which is the temperature used in the analytical $R L C$ plots in figure 3 . The potential height of the sheet below the atoms is approximately $500 \mu \mathrm{K}$, which is effectively infinite when compared to our atom temperatures.

The images were taken by switching on the original near-resonant MOT light for $100 \mu \mathrm{s}$, and collecting the light scattered by the atoms using a single lens, one-to-one imaging system with the image plane on the sensor of our camera. A background image was then subtracted to obtain the final image for any one shot. Eight individual runs were then averaged together for each frame of figure 1 . To count the atom number difference plotted in figure 2 , each imaged was masked off to count the number of atoms in either the left or right container. The error bars in this plot are the standard deviation in the mean for this measurement for the eight runs. Each image was taken after a short (2 ms) time-of-flight for technical reasons.

1. Ramanathan, A. et al. Superflow in a toroidal bose-einstein condensate: An atom circuit with a tunable weak link. Phys. Rev. Lett. 106, 130401 (2011).

2. Seaman, B. T., Kramer, M., Anderson, D. Z. \& Holland, M. J. Atomtronics: Ultracold atom analogs of electronic devices. Phys. Rev. A 75, 023615 (2007)

3. Grimm, R., Weidemüller, M. \& Ovchinnikov, Y. B. Optical dipole traps for neutral atoms. Adv. At. Mol. Opt. Phys. 42, 95-170 (2000).

4. Sharvin, Y. V. On the possible method for studying fermi surfaces. Zh. Eksp. Teor. Fiz. 48, 984 (1965). [Sov. Phys. JETP 21, 655 (1965)].

5. Kadin, A. M. Introduction to Superconducting Circuits (John Wiley and Sons, New York, 1999).

6. Luryi, S. Quantum capacitance devices. Appl. Phys. Lett. 52, 501 (1988).

7. John, D. L., Castro, L. C. \& Pulfrey, D. L. Quantum capacitance in nanoscale device modeling. Appl. Phys. Lett. 96, 5180 (2004).

8. Kittel, C. \& Kroemer, H. Thermal Physics (W. H. Freeman, New York, 1980).
9. van Wees, B. J. et al. Quantized conductance of point contacts in a twodimensional electron gas. Phys. Rev. Lett. 60, 848-850 (1988).

10. Raab, E. L., Prentiss, M., Cable, A., Chu, S. \& Pritchard, D. E. Trapping of neutral sodium atoms with radiation pressure. Phys. Rev. Lett. 59, 2631-2634 (1987).

11. Friedman, N., Kaplan, A., Carasso, D. \& Davidson, N. Observation of chaotic and regular dynamics in atom-optics billiards. Phys. Rev. Lett. 86, 1518-1521 (2001). URL http://link. aps.org/doi/10.1103/PhysRevLett.86.1518.

12. Albiez, M. et al. Direct observation of tunneling and nonlinear self-trapping in a single bosonic josephson junction. Phys. Rev. Lett. 95, 010402 (2005). URL http:// link.aps.org/doi/10. 1103/PhysRevLett.95.010402.

13. Palima, D., Alonzo, C. A., Rodrigo, P. J. \& Glückstad, J. Generalized phase contrast matched to gaussian illumination. Opt. Express 15, 11971-11977 (2007).

\section{Acknowledgments}

The authors would like to thank Bill Phillips, Gretchen Campbell, Kevin Wright, Amy Mathey, Ludwig Mathey and Sergio Muniz for useful discussions during the writing of this letter. This research is funded by the Physical Frontier Center at the Joint Quantum Institute and the Laboratory for Physics Sciences.

\section{Author contributions}

J.G.L., B.J.M. and W.T.H. designed and performed the experiment. J.G.L., C.J.L. and W.T.H. did the theoretical analysis and wrote the manuscript.

\section{Additional information}

Competing financial interests: The authors declare no competing financial interests.

License: This work is licensed under a Creative Commons

Attribution-NonCommercial-NoDerivs 3.0 Unported License. To view a copy of this license, visit http://creativecommons.org/licenses/by-nc-nd/3.0/

How to cite this article: Lee, J.G., McIlvain, B.J., Lobb, C.J. \& Hill, III, W.T. Analogs of Basic Electronic Circuit Elements in a Free-Space Atom Chip. Sci. Rep. 3, 1034; DOI:10.1038/ srep01034 (2013). 